\title{
MORPHOLOGICAL MODELING OF COLD SPRAY COATINGS
}

\author{
Vincent Bortolussi ${ }^{1}$, BRuno Figliuzzi ${ }^{\bowtie, 2}$, FrançOis Willot ${ }^{2}$, MATthieu \\ FAESSEL $^{2}$ AND MICHEL JEANDIN ${ }^{1}$ \\ ${ }^{1}$ Centre for Materials Engineering, Mines ParisTech - PSL Research University, Evry, F-91003, France; ${ }^{2}$ Centre \\ for Mathematical Morphology, Mines ParisTech - PSL Research University, Fontainebleau, 77300, France \\ e-mail: vincent.bortolussi@mines-paristech.fr, bruno.figliuzzi@ mines-paristech.fr, \\ francois.willot@mines-paristech.fr, matthieu.faessel@mines-paristech.fr, michel.jeandin@mines-paristech.fr \\ (Received January 22, 2018; revised March 9, 2018; accepted April 13, 2018)
}

\begin{abstract}
In this article, we study the microstructure of cold sprayed films of copper particles deposited onto a carbon fiber reinforced polymer. The microstructure of the coating is made of a packing of seemingly round-shaped particles of varying sizes embedded in a polymer matrix. The copper particles are separated by thin interstices. The coating is designed to cover the body of recent commercial aircrafts. Its role is to protect the aircraft from lightning impact by ensuring that the surface is conductive enough to evacuate electrical charges. A high resistivity contrast is observed between the copper particles and the polymer matrix. Therefore, the global resistivity of the material is highly dependent on the microstructure geometry.

Following an approach commonly used in materials science, to investigate its influence on the electrical properties of the global material at the macroscopic scale, we design a 3D multiscale stochastic model that enables us to simulate the microstructure. The model is based upon a generalization of the classical JohnsonMehl tessellation, which accounts for the interstices that appear between copper particles. The method is very general and could potentially be applied to model any microstructure exhibiting similar interstices between aggregates of particles.
\end{abstract}

Keywords: cold spray, mathematical morphology, microstructure simulation, stereology, stochastic geometry.

\section{INTRODUCTION}

Modern materials manufacturing has evolved toward a better control and optimization at the microscopic scale. Hence, microstructures commonly exhibit complex morphologies mixing various materials including carbon fibers arrangements into polymer matrix. The physical and mechanical features of heterogeneous materials at the macroscopic scale are largely dictated by phenomena appearing at the microscopic level. Therefore, the development of numerical tools deriving the physical properties of a material at the macroscopic scale from its geometry at the microscopic scale has been a very active topic of research over the past few years (Torquato, 2002).

Current computational capabilities and imaging techniques can fill the gap between microstructure observation and computation of effective physical and mechanical properties through simulation. However, if recent tomography processes can entirely recreate complex microstructures, some materials remains inadapted to imaging process involving high energy beams. In this case, an alternative is to rely on stochastic models to describe and simulate microstructures. Computational tools and models brought by mathematical morphology (Serra, 1982; Soille, 2003) allow to generate and simulate complex microstructures on which one can perform physical simulations (Zeng et al., 2008). This method can cope very well with multiscale and multiphased random sets (Jeulin, 2012), as demonstrated by recent studies investigating the effects of rigid fillers into soft matrix such as black carbon particles embedded into a polymer matrix (Jean et al., 2011; Figliuzzi et al., 2016) or shells of argan nut in polypropylene (El Moumen et al., 2014). Recently, similar methods were used to describe polycristalyne microstructures (Gasnier et al., 2015).

In this study, our aim is to simulate the electrical properties of a biphased coating obtained by thermal spraying. The coating is designed to cover the body of recent commercial aircrafts. Its role is to protect the aircraft from lightning impact by ensuring that the surface is conductive enough to evacuate electrical charges. Numerous physical and mechanical properties of cold sprayed coatings have already been investigated in the literature, including oxidation (Cochelin et al., 1999), Young's modulus (Amsellem et al., 2008), porosity (Beauvais et al., 2008) or thermal conductivity (Bobzin et al., 2012). Jeandin et al. (1999) presented a model of coating build up in plasma spray. More recently, Delloro et al. (2017) developed a morphological model for the cold spray process that accounts for 
the morphology of the deposit and for mechanical, physical and dynamic phenomena. However, to our knowledge, this study constitutes one of the first attempt to model the electrical properties of cold spray deposits from their microstructure.

In this paper we present a 3D stochastic model describing the coating microstructure. The model is derived from experimental images of slices of the material taken with a microscope. The microstructure is constituted of copper particles embedded in a PEEK polymer matrix. The particles are separated by thin interstices of PEEK polymer. In terms of resistivity, a high constrast prevails between the polymer and the copper particles. Hence, a key issue toward an effective modeling of the microstructure is to accurately reproduce the interstice thicknesses and repartition. To that end, we introduce a new stochastic model derived from the classical Johnson-Mehl tessellation (Johnson and Mehl, 1939; Møller, 1989; 1992; 1994) that enables us to properly reproduce these geometrical characteristics. The method is very general and could potentially be applied to model any microstructure exhibiting similar interstices between aggregates of particles.

The article is organized as follows. In section "Materials", we describe the materials on which we conduct our study and we briefly recall the basics of cold spray deposition. In section "Image segmentation and characterization", we discuss the segmentation techniques and the statistical features used to process the experimental microscopic images. The 3D stochastic model is described in section "Stochastic model for the microstructure", and we comment on the results of the study in section "Results and discussion". Conclusions are drawn in the last section.

\section{MATERIALS}

\section{MATERIALS}

The body of recent commercial aircrafts is made of carbon fiber-reinforced polymers. These materials ensure high mechanical properties while being lighter than regular aluminium alloys. However, the polymer matrix is generally highly electrically insulating, which causes security concerns with lightning. Highergrade aerospace composites are made with a matrix of PEEK (Poly-Ether-Ether-Ketone), a thermoplastic polymer offering good mechanical and thermal properties. In addition the PEEK is a very good insulator with a resistivity of $1.0 \times 10^{14} \Omega \mathrm{m}$.
To evacuate electrical charges in case of lightning impact, a mesh of copper is ordinary applied onto the composite body. Copper is a readily available electrical conductor, that is easily machinable and corrosion resistant. The electrical resistivity of copper is $1.68 \times 10^{-8} \Omega \mathrm{m}$.

To avoid complex manufacturing and assembly of copper meshings, we developed a new coating method which relies on copper powder thermally sprayed onto composite parts. More precisely, to achieve an adherent and electrically conductive layer we use a powders mixture. The mixture contains $80 \%$ volumetric of spherical copper powder $(10-35 \mu \mathrm{m})$ and $20 \%$ of irregular PEEK particles $(35-65 \mu \mathrm{m})$, increasing adherence with the composite. The mixture is sprayed using the cold-gas dynamic spraying or "cold spray" process.

\section{PROCESS}

Cold spray is a thermal spraying process using high speed spraying of powders to create a dense coating. It relies on a gas flow to drive the powder toward the substrate. The gas is usually nitrogen under pressure (1 to $4 \mathrm{MPa}$ ), which is heated (200 to $800^{\circ} \mathrm{C}$ ) and accelerated through a convergentdivergent (De Laval type) nozzle. The powder is injected in the accelerated gas flow and can reach supersonic speed. Due to relatively low spraying temperature and processing time, powder particles remain solid. When powder particles impact the substrate, both of them undergo plastic strain. The adhesion of the coating is usually guaranteed by mechanical and chemical bonding. This process can lead to very dense coating due to dynamic impact, as well as low oxide content due to low temperature. In this study, we use manufactured coating made of a copper and PEEK mixture to investigate the microstructure of cold spray coatings.

\section{Samples preparation}

Cold spray coatings were cross-sectioned and polished previous to observation. Coating samples were cut in two directions, along the spraying path and orthogonal to the spraying path. Cutting and polishing debond copper particles due to poor mechanical anchorage in the matrix, leaving dark holes at the surface. Manual polishing severely influences debonding phenomenon. Samples were then metallized with a $2 \mathrm{~nm}$ layer of gold-palladium using a Cressington sputter coater. This is a key operation as the layer modifies the colour of the PEEK matrix, by greatly enhancing colour gradient between phases. We observed cross-sections at $\times 20$ magnification in bright field using a Leica optical microscope with 
a resolution of $0.2428 \mu \mathrm{m}$ per pixel. The selected observation scale was chosen to obtain a representative fraction of copper while highlighting PEEK interstices.

\section{Microstructure}

The microstructure of the coating can be observed in Fig. 1 with copper particles in yellow, dark footprints and a grey matrix of PEEK. The resulting cold spray coating contains copper particles embedded in a PEEK matrix. The matrix is obtained from irregular PEEK particles highly deformed upon high speed impact. It is considered dense with no pores appearing at this scale. Copper particles are deforming solely when impacting each other resulting in a limited plastic strain. These particles are forming a network of copper clusters.

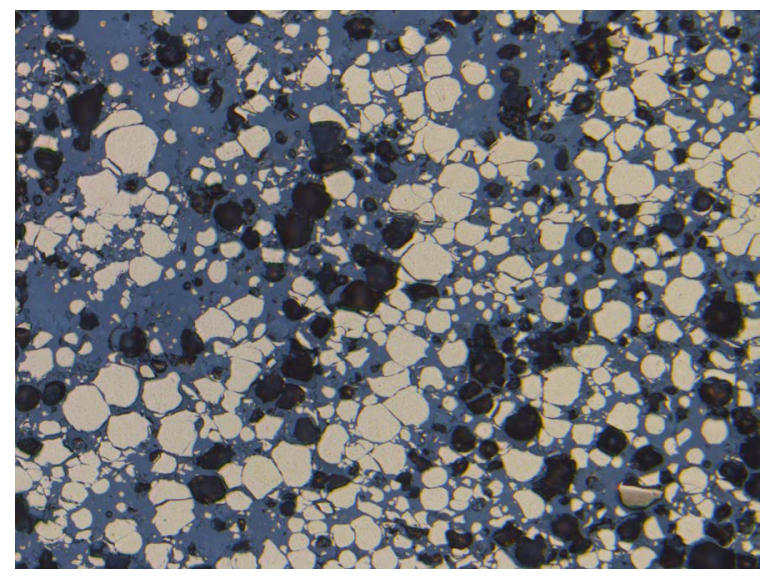

Fig. 1: Optical microscope cross-section of the coating microstructure with debonded particles in black $\left(2560 \times 1920\right.$ pixels equals to $\left.620 \times 476 \mu \mathrm{m}^{2}\right)$.

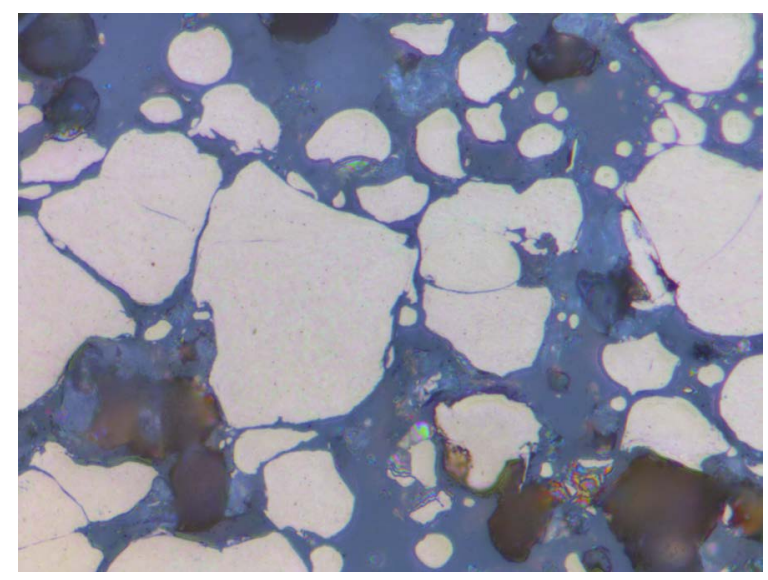

Fig. 2: Magnified optical microscope cross-section of the microstructure of the coating.
Common cold spraying of metal particles onto metal substrate generally involve chemical bonding and interdiffusion at the interfaces between particles and substrate. In our case, optical observation at larger scale highlights thin $(<10 \mu \mathrm{m})$ PEEK layers lying between deformed copper particles, as shown in Fig. 2. These interstices prevent direct contact between copper particles. Filled with electrically insulating PEEK, they allegedly increase resistivity, thus lowering the coating conductivity.

\section{IMAGE SEGMENTATION AND CHARACTERIZATION}

In this section, we describe the segmentation process developed for the images of the microstructure of the coating.

\section{SEGMENTATION PROCESS}

The segmentation process is performed with a Python script based upon the free image library SMIL (Faessel and Bilodeau, 2014). It aims at identifying the two phases of the material, namely the PEEK matrix and the copper aggregates. Previous to any segmentation operation, we transform the RGBimages from the microscope into 8-bits images, by extracting the second channel, as shown in Fig. 3a. Then, we threshold the image with an automatic Otsu process (Otsu, 1975) involving two thresholds. This leads to three-phased coloured images, as shown in Fig. 3b, where copper particles appear in yellow, footprints of the debonded particles in black and the PEEK matrix in red. Due to low greylevel gradient, this first thresholding operation is not able to separate all close particles.

In a second step, we merge the footprints into the matrix by thresholding the particles (Fig. 3c). Next, small isolated particles and observation artefacts with an area smaller than 100 pixels are removed. Then we select inner markers for the particles with by keeping the $h$-maxima of a distance function applied on the particles. This operation can be seen on Fig. $3 d$ and the result on Fig. 3e.

Once the markers are selected, particles are separated with a watershed process (Beucher and Lantuéjoul, 1979; Vincent and Soille, 1991), using the core of the particles and a grey scale colour gradient map to define the boundaries of each cell. Separation achieved with the watershed process is assessed on Fig. 3f. Finally we label each particles obtained after the watershed. The watershed process is able to achieve good separation of close particles, even if the interstices do not always 


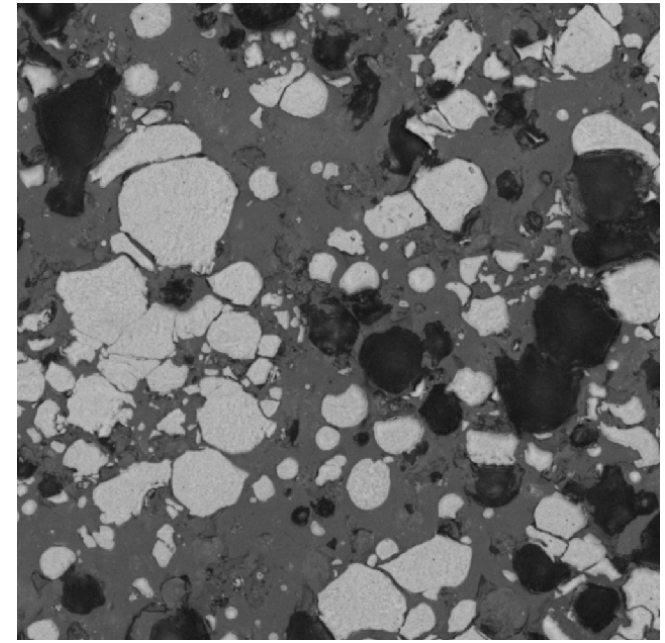

(a)

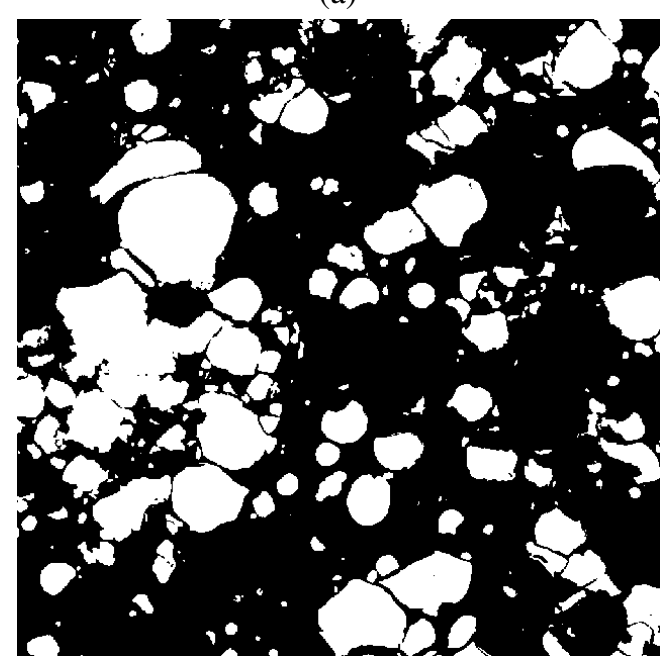

(c)

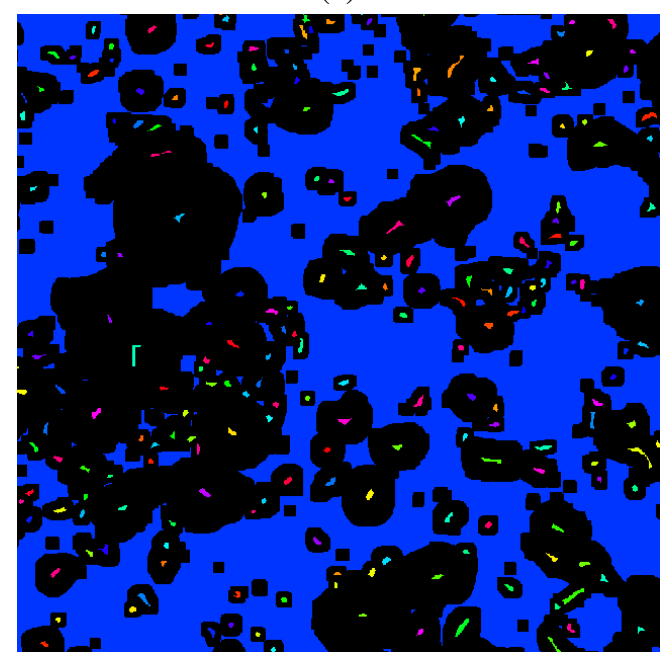

(e)

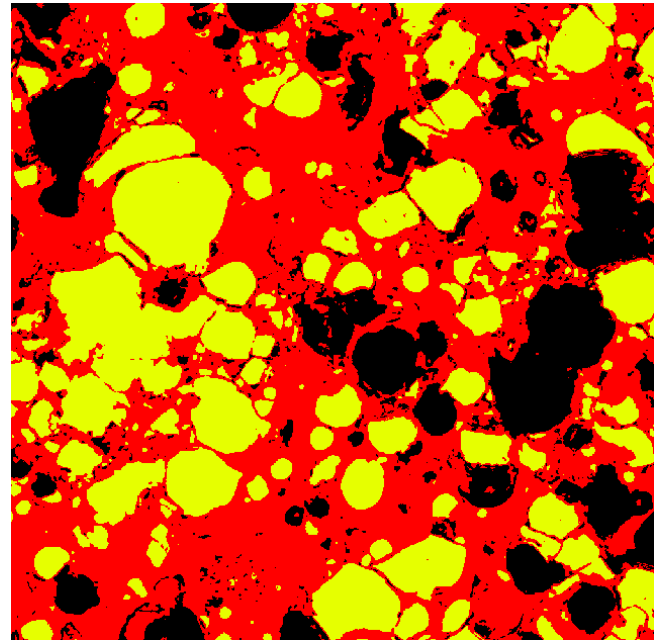

(b)

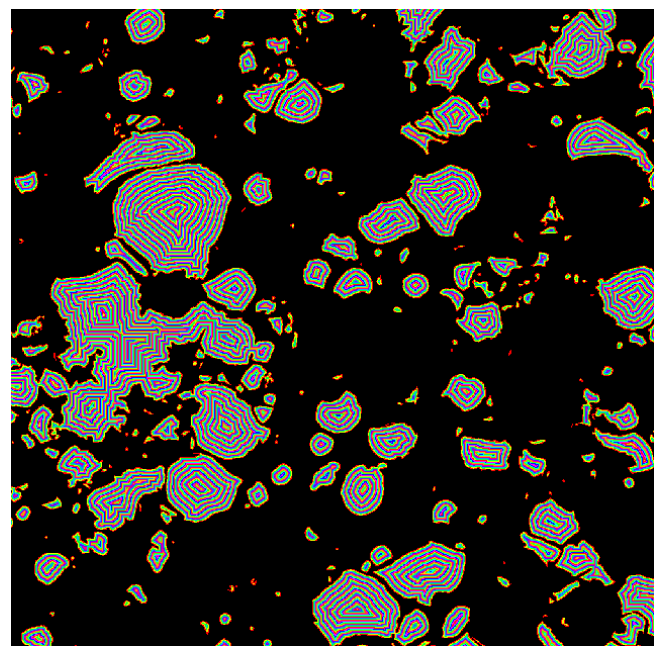

(d)

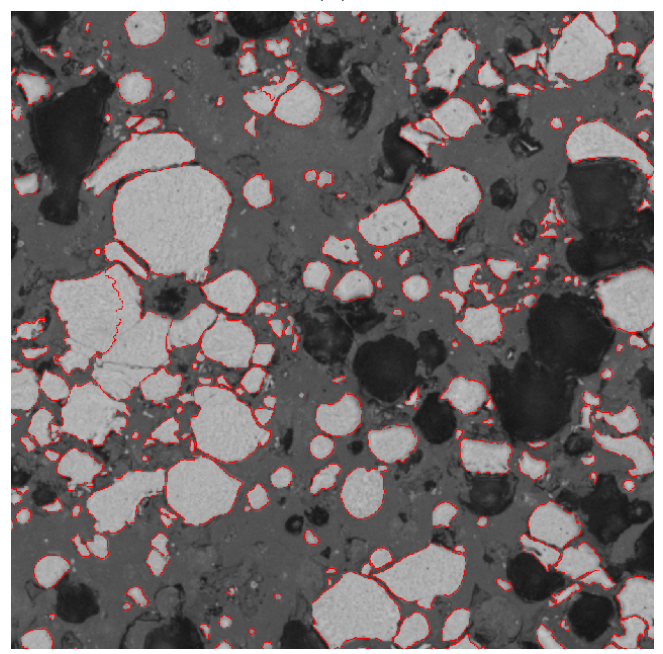

(f)

Fig. 3: Segmentation process of particles. (a) Grey scale image. (b) Thresholded image. (c) Particles selection. (d) Distance function on particles. (e) Cores of particles. (f) Watershed procedure on particles. 


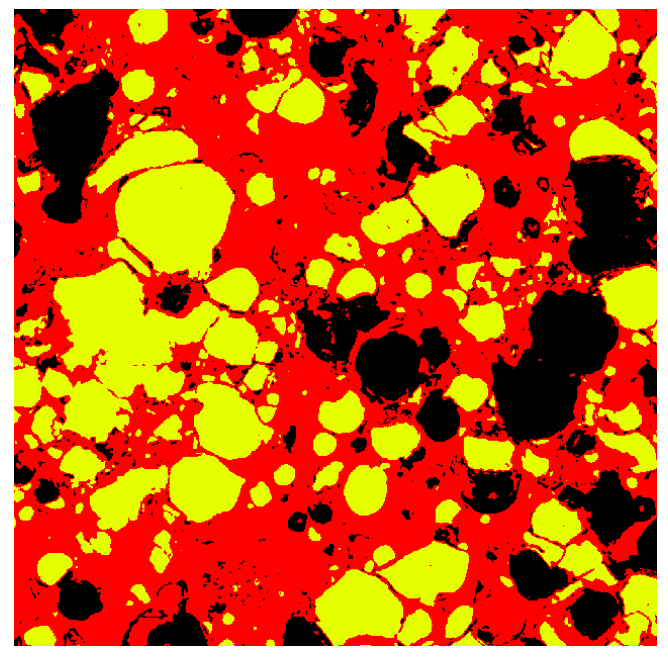

(a)

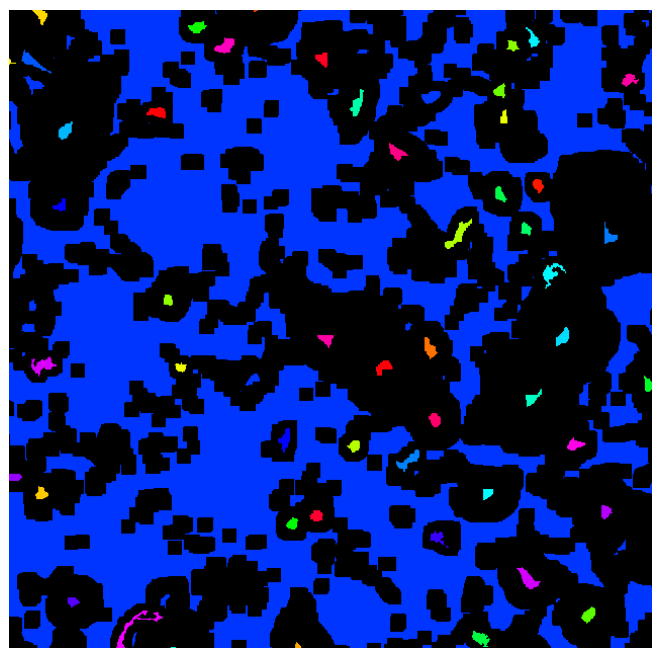

(c)

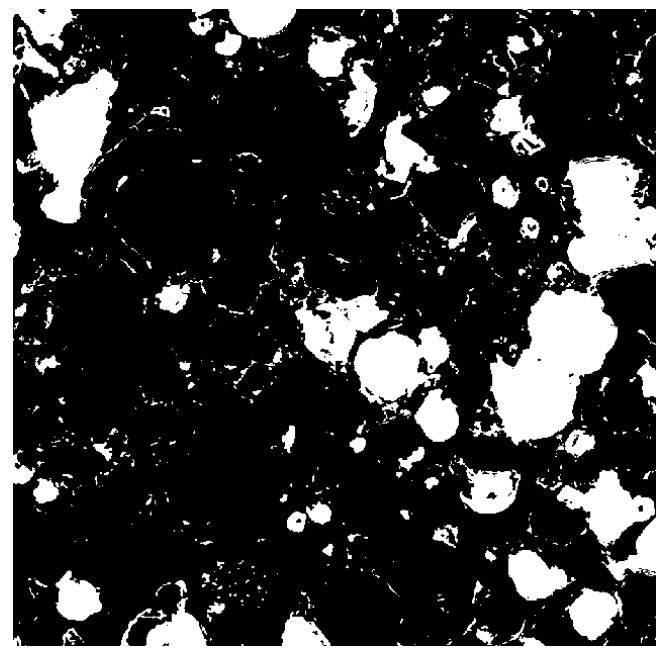

(b)

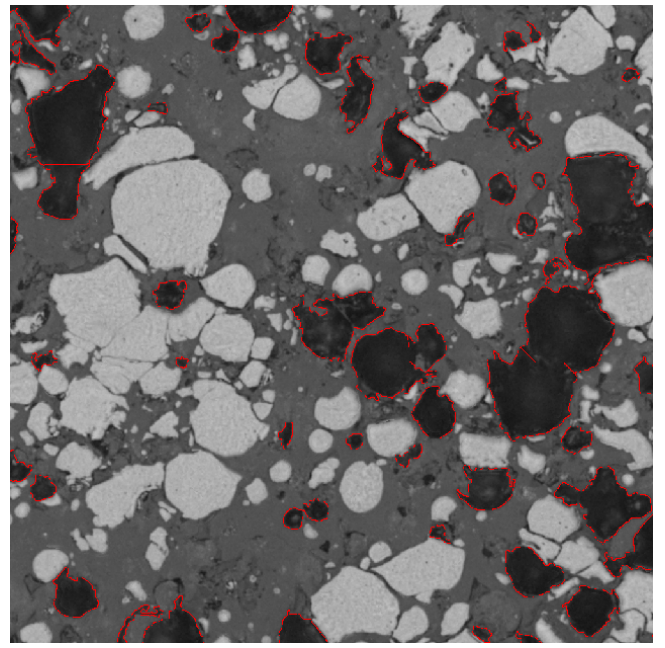

(d)

Fig. 4: Segmentation process of debonded particles. (a) Thresholded image. (b) Debonded particles selection. (c) Cores of debonded particles. (d) Watershed procedure on debonded particles.

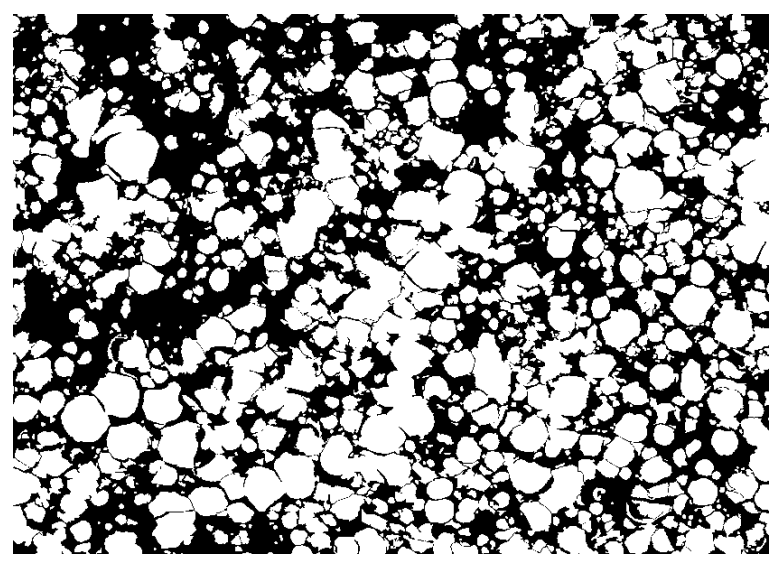

(a)

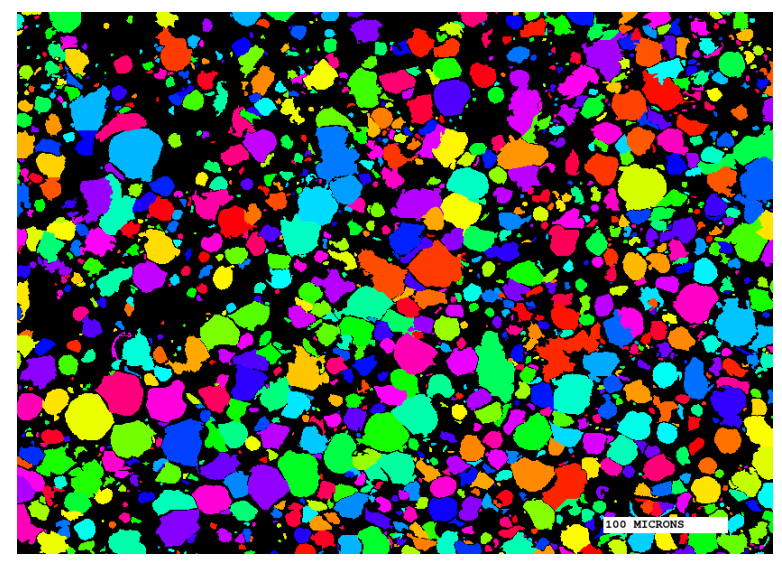

(b)

Fig. 5: (a) Final binary image. (b) Labelled particles. 
appear on thresholded images. The main steps depicted on Fig. 3 use smaller images than the real samples.

The same exact process is applied on debonded particles. This time real particles are merged into the matrix, and footprints are filled by changing pixels values of thresholded images (Fig. 4a and Fig. 4b). As one footprint could have contained several particles, this filling process is not able to reconstruct hypothetical interstices separating them. Then we apply the segmentation process used on the particles to label the debonded particles, as shown in Fig. $4 \mathrm{c}$ and Fig. 4 d.

Finally we superimpose the two labelled images (Fig. 5b). By colouring the matrix in black and each particle in white we obtain a binary image like Fig. 5a, used for morphological measurements. The segmentation process was applied on 13 randomlyselected images of $2560 \times 1960$ pixels representing an area of $620 \mu \mathrm{m} \times 476 \mu \mathrm{m}$.

\section{MORPHOLOGICAL DATA}

In this section, we conduct morphological measurements on the binary images to characterize the spatial distribution of copper particles. We focus more specifically on three morphological descriptions: the covariance, the granulometry and the thicknesses distribution of the interstices. These morphological measurements will be further exploited to compute the parameters of the microstructure model.

\section{Covariance}

The covariance is defined as the probability:

$$
C(r)=P\{x \in \mathscr{H}, x+r \in \mathscr{H}\},
$$

where $\mathscr{H}$ is the union of copper particles in the coating, $x$ some point, and $r$ some vector in $\mathbb{R}^{2}$.

For a stationary random set $\mathscr{H}$, the covariance does not depends on $x$ anymore but only on $r$. It depends on the orientation and on the modulus of $r$ and represents the probability for two points separated by distance $r$ to belong to the same phase of the material. For $r=0$, the covariance is equal to the surface fraction of $\mathscr{H}$ (in 2D). At large distance $|r| \gg 1$ the two events $x \in \mathscr{H}$ and $x+r \in \mathscr{H}$ become uncorrelated and $C(r=$ $\infty) \approx C(0)^{2}$.

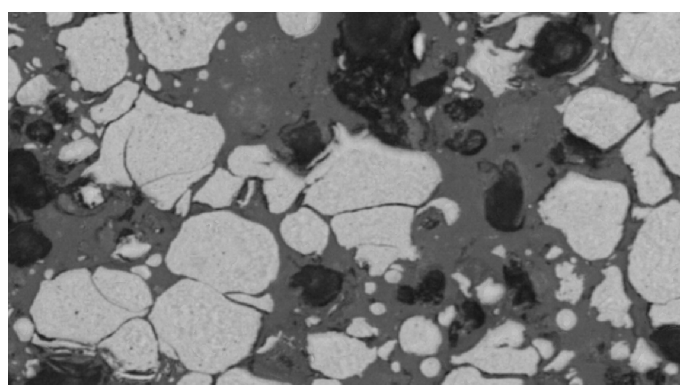

(a)

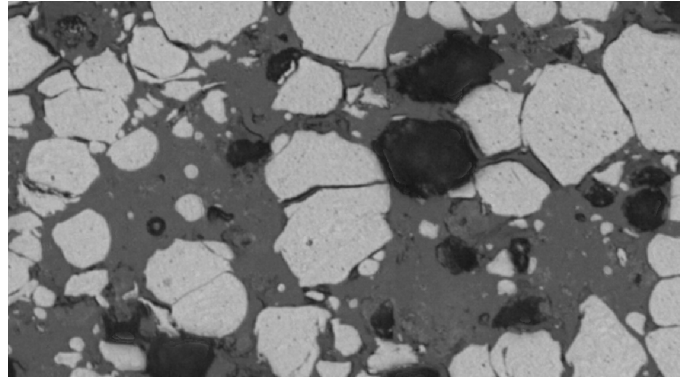

(b)

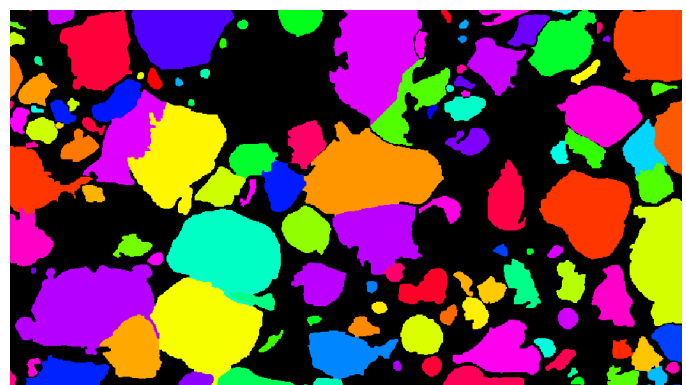

(c)

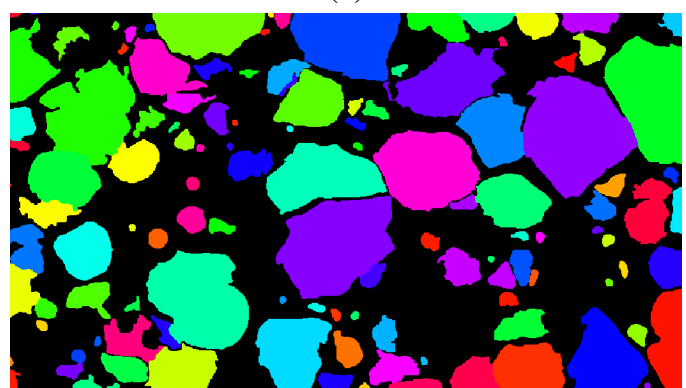

(d)

Fig. 6: Separation of close particles. (a) and (b) Examples of close particles. (c) Separated particles without interstices. (d) Separated particles with interstices.

In this study, the covariance is measured for vectors of increasing moduli along the horizontal and vertical directions. Note that if the microstructure is isotropic, the covariance does not depend on the orientation of $r$. In our case, isotropy and stationarity have been assessed using all sample images. Fig. 7 shows the covariances measured on the segmented image represented in Fig. 1. 


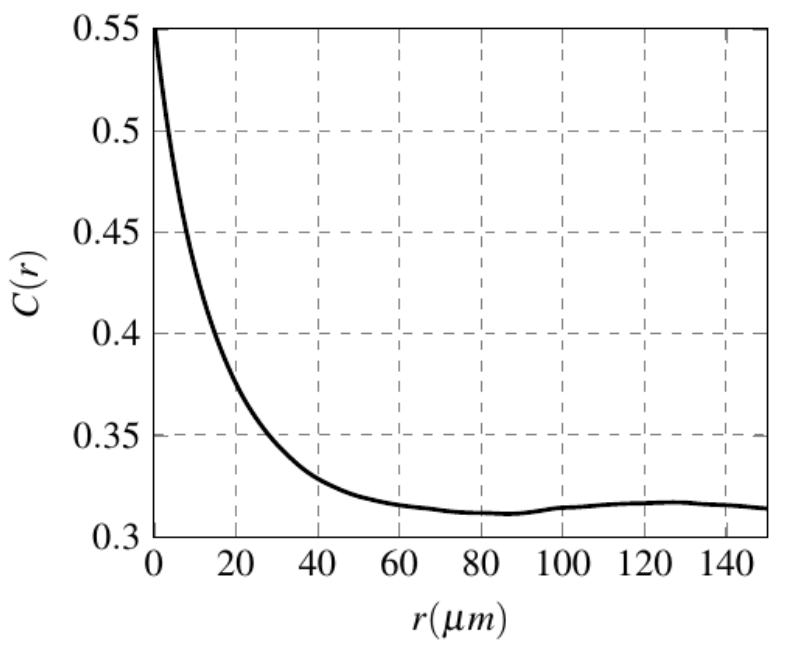

Fig. 7: Covariance curve as measured on the microstructure displayed in Fig. 5a.

The covariances obtained on the sample microstructure images show small dispersion, with a mean surface fraction of $55 \%$ and a standard deviation of $4.9 \%$. We compared the mean covariances measured vertically, horizontally and diagonally on the segmented images.

\section{Granulometry}

The cumulative granulometry is calculated by opening the copper phase with probability

$$
G(s)=\frac{P\{x \in \mathscr{H}\}-P\{x \in \mathscr{H}(S ; s)\}}{P\{x \in \mathscr{H}\}},
$$

where $x$ is a point in the image, $\mathscr{H}$ is the copper phase and $\mathscr{H}(S ; s)$ is the morphological opening of $\mathscr{H}$ by a structural element $S$ dilated by size $s$. In this case the structuring element is a 8-connexity square. The cumulative granulometry is measured on each image, giving a mean granulometry. However due to limited interstices segmentation, the measured granulometry does not completely reflect the real granulometry of the microstructure. In addition, it is important to note that the latter is different from the initial powder's granulometry because of the spraying process: some of the particles bounce at impact, thus missing in the microstructure. In addition, particules deform upon impact.

Fig. 8 shows the granulometry as measured on the segmented image shown in Fig. 1. The mean cumulative granulometry shows that $10 \%$ of the microstructure is removed after an opening of size $1.7 \mu \mathrm{m}$ ( $\simeq 7$ pixels $), 50 \%$ of the microstructure is

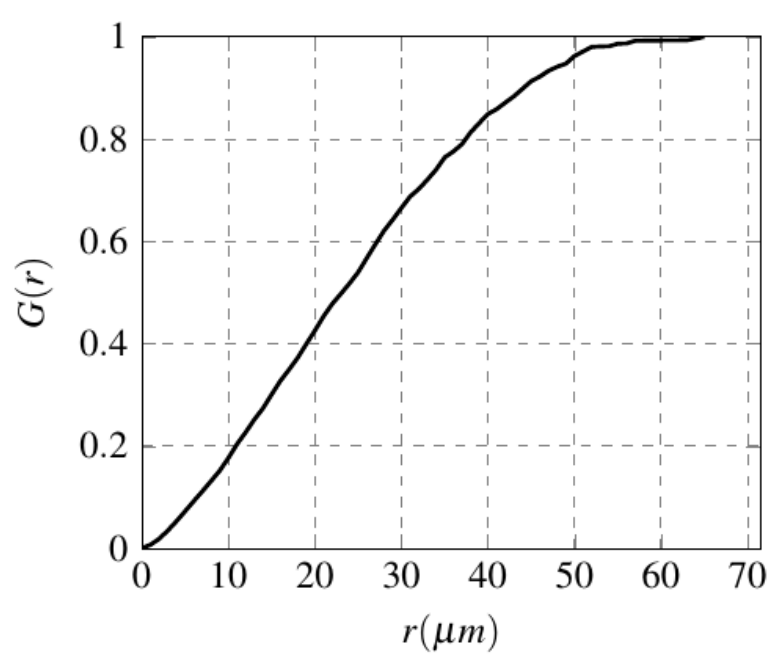

Fig. 8: Granulometry curve as measured on Fig. 5a.

removed after an opening of size $5.7 \mu \mathrm{m}(\simeq 24$ pixels $)$ and $90 \%$ of the microstructure is removed after an opening of size $10.6 \mu \mathrm{m}$ ( $\simeq 44$ pixels). When measured on the thirteen samples, it is less dispersed compared to the covariance.

\section{Interstice thickness}

Interstices appearing between copper particles are a key feature of the microstructure that strongly impact the conductivity of the material at the macroscopic scale. Therefore, they must be carefully described.

The labelled image issued from the segmentation process enables us to determine the number of nonconnected particles $n_{0}$. After a dilation of 1 pixel, subtraction of the number of remaining isolated particles $n_{1}$ to the initial quantity gives the number of particles separated by a distance of 2 pixel: $n_{\text {interstice }}=$ $n_{0}-n_{1}$. Actually this process only measure distance of an even number of pixels. This method is repeated for 20 dilations on all sample images, thus yielding the size distribution of the interstices thickness.

We apply the interstices measuring process to obtain the thickness distribution displayed in Fig. 9.

The average distribution is fitted with an exponential law. The probability density function of this distribution is:

$$
p(x, k)=k e^{-x k} .
$$

The exponential law with $k=1.19 \mu \mathrm{m}^{-1}$ displayed in Fig. 10 shows good agreement with the experimental measurements. Interstices thickness were also measured on magnified images, showing similar distribution. 


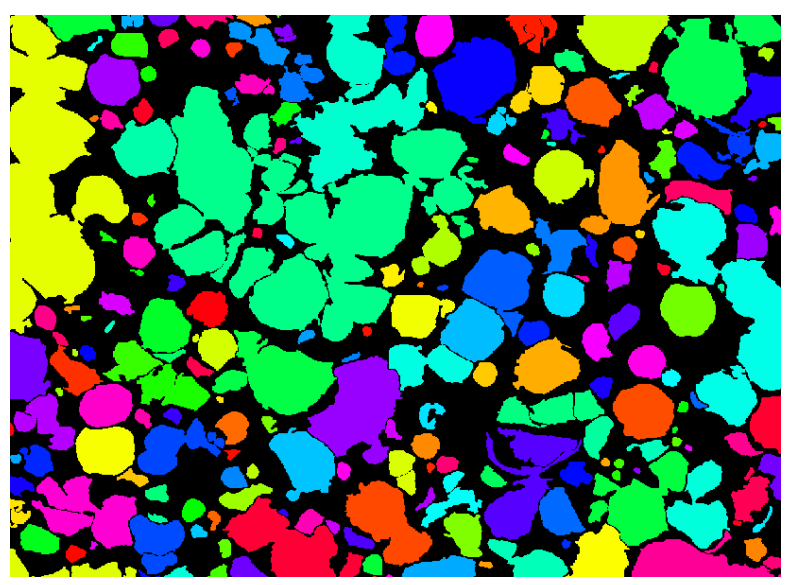

(a)

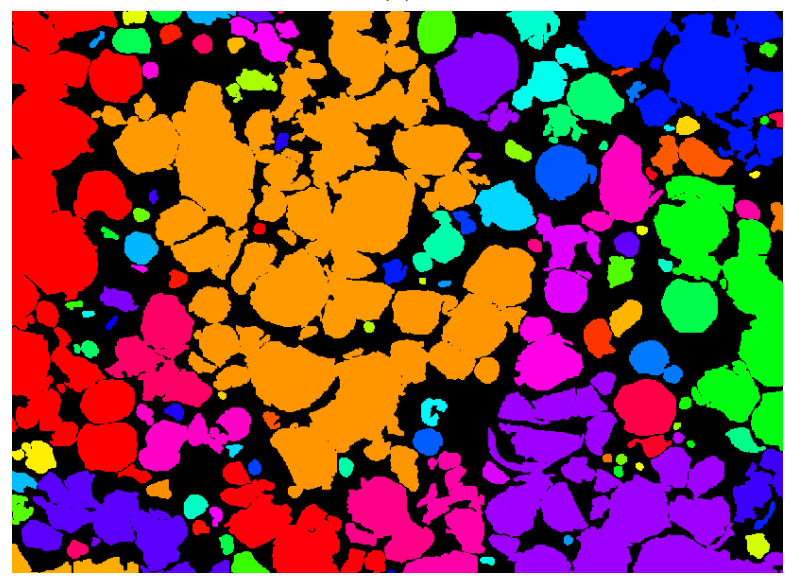

(b)

Fig. 9: Interstices measurement process on a quarter of an image. (a) Initial labelled particles. (b) Labelled particles after 2 dilation.

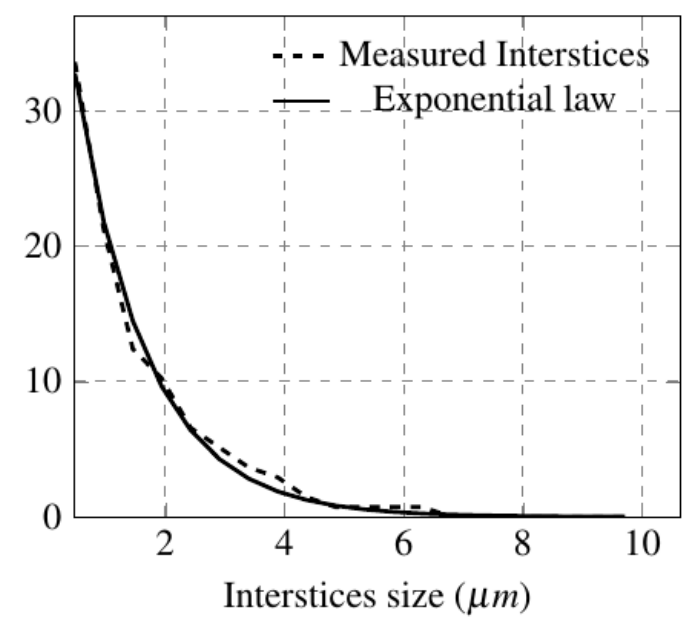

Fig. 10: Interstices mean thickness distribution.

\section{Copper phase perimeter and area}

The perimeter of the copper phase can be roughly estimated by eroding the copper particles using a 8-connexity square of size one and considering the residue from the initial copper particles. Enclosure of the particles remains with a thickness of one pixel. An area measurement of the enclosure yields the perimeter of the particles per unit area. The perimeter is very sensitive to the segmentation of the interstices. The area is equal to $C(0), C$ being the covariance.

For experimental samples that have been cut in directions parallel to the covered substrate, the measurements yield a mean perimeter per unit area of $0.19 \mu^{-1}$ for the copper phase with a standard deviation of $0.01 \mu \mathrm{m}^{-1}$. The mean area of the copper phase is 0.55 with a standard deviation of 0.056 . For experimental samples that have been cut in the vertical direction, the measurements yield a mean area for the copper phase of 0.59 with a standard deviation of 0.047 .

\section{STOCHASTIC MODEL FOR THE MICROSTRUCTURE}

In this section, we introduce a probabilistic model for the 3D microstructures of the coating. We assume that the microstructure is described by copper spheres embedded in a PEEK matrix and separated by thin PEEK interstices. Covariance, copper fraction, granulometry, and interstice thickness are measured on 2D slices of the coating, thus 3D model's parameters must be inferred from these $2 \mathrm{D}$ informations. The final two-scale model relies on a two-step simulation process, namely 1/ Boolean spheres implantation of intensity $\theta$, whose radii follow a Gamma distribution law with parameters $\lambda$ and $a$; and $2 /$ Interstices implantation based on a modified Johnson-Mehl tessellation.

The boolean spheres represent copper particles, forming clusters due to interpenetration. Simulated volumes are sliced in $1000 \times 1000$ pixels images.

\section{BOOLEAN MODEL OF SPHERES}

We assume that the distribution of radii of the spheres is described by a Gamma law. The probability density function of the Gamma law is given by

$$
p(r, \lambda, a)=\frac{r^{a-1}}{\Gamma(a) \lambda^{a}} \exp \left(-\frac{r}{\lambda}\right),
$$

where $\Gamma$ denotes the Gamma function. The average radius of the spheres is $a \lambda$. Its variance is $a \lambda^{2}$.

We can easily show that the average surface of a grain is 


$$
S_{v}=\int_{0}^{+\infty} \frac{4 \pi r^{a+1}}{\Gamma(a) \lambda^{a}} \exp \left(-\frac{r}{\lambda}\right) \mathrm{d} r=4 \pi \lambda^{2} a(a+1)
$$

Similarly, the average volume of a grain is

$$
\begin{aligned}
V_{v} & =\int_{0}^{+\infty} \frac{4 \pi r^{a+2}}{3 \Gamma(a) \lambda^{a}} \exp \left(-\frac{r}{\lambda}\right) \mathrm{d} r \\
& =\frac{4 \pi}{3} \pi \lambda^{3} a(a+1)(a+2) .
\end{aligned}
$$

Let us denote by $\theta$ the intensity of the 3D Boolean model of spheres, and by $\theta_{2}$ the intensity of the corresponding Boolean model of disk as observed on 2D slices extracted from the original model. To determine the 3D parameters of the model from the 2D measurements, we use the stereological formulae

$$
\theta V_{v}=\theta_{2} \bar{A}
$$

and

$$
\theta S_{v}=\frac{4}{\pi} \theta_{2} \bar{L}
$$

where $\bar{A}$ is the mean area of the sliced spheres and $\bar{L}$ their perimeter. We need to link the 2D measurements to the parameters of the boolean model. To that end, we rely on Miles' formulae (Miles, 1972; Chiu et al., 2013; Schneider and Weil, 2008)

$$
\mathscr{A}_{A}=1-e^{\left(-\theta_{2} \bar{A}\right)},
$$

and

$$
\mathscr{L}_{A}=\theta_{2} \bar{L}\left(1-\mathscr{A}_{A}\right),
$$

where $\mathscr{A}_{a}$ is the mean surface fraction of copper on the segmented images and $\mathscr{L}_{A}$ is the mean perimeter of the copper phase on the segmented images divided by the total surface.

Using Miles' formulae in conjunction with stereological formulae (Chiu et al., 2013; Schneider and Weil, 2008), we find, for the Boolean model

$$
\mathscr{A}_{A}=1-\exp \left(-\theta V_{v}\right),
$$

and

$$
\mathscr{L}_{A}=\frac{\pi}{4} \theta S_{V} \exp \left(-\theta V_{v}\right) .
$$

Overall, we have three unknowns in these equations, namely the intensity $\theta$ of the Boolean model and the parameters $a$ and $\lambda$ of the Gamma distribution. Hence, we can express all parameters as functions of $a$. Using Eqs. 11 and 12, we find

$$
\theta=-\frac{3}{4 \pi \lambda^{3} a(a+1)(a+2)} \ln \left(1-\mathscr{A}_{A}\right),
$$

and

$$
\lambda=-\frac{3 \pi}{4(a+2) \mathscr{L}_{A}}\left(1-\mathscr{A}_{A}\right) \ln \left(1-\mathscr{A}_{A}\right) .
$$

To determine the parameters of the stochastic model, we rely on a maximum likehood approach to find the parameters that minimize the least-square distance between the covariance of the simulated microstructure and the covariance that is measured on the available experimental dataset. However $\mathscr{L}_{A}$ is highly influenced by interconnection between particles. As many particles remains in contact due to poor interstices segmentation, computing $\theta$ only from $\lambda$ and $a$ provides a more robust algorithm.

\section{INTERSTICES IMPLANTATION}

To simulate the second scale of the microstructure, that corresponds to the interstices between the particles of the same aggregate, we rely on random JohnsonMehl tessellations restricted to each aggregate, or connected component of the first scale of the microstructure.

Let $\Omega$ denote a given volume in $\mathbb{R}^{3}$. A Voronoï tessellation is a tessellation built from a Poisson point process $\mathscr{P}$ in the space $\mathbb{R}^{3}$. Every point $x$ of $\mathbb{R}^{3}$ is associated to the class $\mathscr{C}_{i}$ containing all points of $\mathbb{R}^{3}$ closer from the point $x_{i}$ of $\mathscr{P}$ than from any other point of $\mathscr{P}$. Hence, the classes $C_{i}, i=1, . ., N$ are defined by

$$
C_{i}=\left\{y \in \mathbb{R}^{3}, \forall j \neq i,\left\|x_{i}-y\right\| \leq\left\|x_{j}-y\right\|\right\} .
$$

With probability one, Voronoï tessellations are normal and face-to-face. Voronoï tessellations are characterized by one single parameter, namely the intensity of the underlying point process.

The Johnson-Mehl tessellation is a sequential version of the Voronoï model (Johnson and Mehl, 1939; Møller, 1989; 1992; 1994), where the Poisson points are implanted sequentially according to a parameter $t$ which can conveniently be interpreted as an implantation time. All classes grow then isotropically with the same rate $v$, and the growth of crystal boundaries is stopped when they meet. All Poisson points falling in an existing cell are removed. From a mathematical perspective, a Johnson-Mehl tessellation is constructed from a sequential Poisson point process where the points $x_{i}, i=1, \ldots, N$ are implanted sequentially at a time $t_{i}, i=1, \ldots, N$. The classes $C_{i}, i=1, \ldots, N$ corresponding to the points $x_{i}, i=1, \ldots, N$ are defined by

$$
C_{i}=\left\{y \in \mathbb{R}^{3}, \forall j \neq i, t_{i}+\frac{\left\|x_{i}-y\right\|}{v} \leq t_{j}+\frac{\left\|x_{j}-y\right\|}{v}\right\} .
$$


Note that when all times are set to zero, we recover the classical Poisson-Voronoï tessellation model.

In this study, to account for the interstices between grains of the same aggregate, we rely on a modified version of the Johnson-Mehl tessellation For each grain $n$, we simulate a random number $\zeta_{n}$ according to an exponential law with some mean $k$. The classes are now defined, for a given aggregate $\mathscr{A}$, by

$$
C_{i}=\left\{y \in \mathscr{A}, \forall j \neq i, t_{i}+\frac{\left\|x_{i}-y\right\|}{v}+\zeta_{i} \leq t_{j}+\frac{\left\|x_{j}-y\right\|}{v}\right\},
$$

where \|\| is the geodesic distance on the set $\mathscr{B}$ defined by the first scale of the model. With this definition, we note that some points of the aggregates don't belong to any class of the Johnson-Mehl tessellation. We consider that these points form the interstices between the grains of the microstructure.

The main challenge faced using Johnson-Mehl tesselation was to quickly calculate the position of the interstices. To do so we rely on the fast marching method in 2D or in 3D (Sethian, 1996). This method solves boundary value problems of the eikonal equation in the spatial domain $\Omega$ :

$$
\begin{array}{rlrl}
|\nabla u(x)| & =\frac{1}{f(x)}, & & \text { for } x \in \Omega, \\
u(x) & =0, & \text { for } x \in \partial \Omega,
\end{array}
$$

in which $u(x)$ is the arrival time of the front and $f(x)$ its propagation time. This method allows to quickly compute propagation time of closed curves from nuclei in space.

A question remains, which is how to select the initial nuclei of the tessellation and the nucleation times. While selecting the nuclei, our aim is to preserve the geometrical shape of the grains of the microstructure. The goal is to set the nuclei in the center of connected components to simulate a granulary microstructure. Therefore, we rely on the $h$-maxima of the distance function to generate the nuclei. The $h$-maxima of the distance function form connected components. For each component, we select its barycentre to be the location of a nucleus. The threshold for the $h$-maxima is selected after an optimization procedure that aims at minimizing the distance between the granulometries. In addition, we only keep a proportion $p$ of the $h$-maxima, $p$ being an additional parameter of the model. For each nucleus $n$, we denote by $d_{n}$ the value of the distance function at the location of the nucleus. The nucleation time associated to nucleus $n$ is defined to be

$$
t_{n}=\max _{m} d_{m}-d_{n} .
$$

\section{MAXIMUM LIKEHOOD FOR PARAMETERS ESTIMATION}

The parameters estimation follows a two-step process. For estimating the parameters $a$ and $\lambda$ of the first scale of the model, we compute a slice of a 3D microstructure resulting from the Boolean model. We use $1000 \times 1000$ pixels slices, taken from a larger volume to account for border effects. The spheres are implanted within this given volume, requiring it to be large enough to reach a representative copper fraction. We measure then the covariance associated to the slices. The covariance is compared with the experimental one. We aim at minimizing the least square distance between both covariances. To do so, we rely on a Nelder-Mead procedure optimizing on the parameters of the radii distribution: $a$ and $\lambda$. Each guess of these parameters gives a value for $\theta$ according to relation (Eq. 13). With good initial values, it takes less than 40 iterations to fit the experimental covariance.

For the second scale of the model, we rely on a similar procedure, this time involving the granulometry measurements, to estimate the threshold value $h$ of the $h$-maxima distance that is used to select the nuclei locations, the proportion $p$ of $h$-maxima that needs to be removed and the parameter of the exponential law describing interstice thickness.

\section{NUMERICAL IMPLEMENTATION OF THE MODEL}

In this section, we briefly recall the main steps of the developed algorithm and we describe the numerical implementation of the two-scale model. This implementation aims at simulating 3D microstructures while reducing calculation time. The algorithm proceeds as follows:

1. The first step of the algorithm is to simulate a Boolean model of spheres with intensity $\theta$. The radii of the implanted spheres follow the Gamma law (Eq. 4). The boolean model of spheres is simulated using the 3D vectorial VTKbased library vtkSim (Faessel, 2016; Faessel and Jeulin, 2011). To accelerate volume building from simulation, building was performed using subvolumes. This method allows to simulate $500 \times 500 \times 500$ voxels microstructures in $400 \mathrm{~s}$ (proc time) on a PC (Ubuntu 6.14 i7 $2.20 \mathrm{GHz}$ 6 GB RAM). The complexity of the algorithm is $O\left(N^{2}\right)$ with $N$ the number of voxels in the volume.

2. The second step of the algorithm is the simulation of the interstices. The algorithm follows the following steps. 
(a) Calculation of the distance function for the microstructure obtained from the Boolean model of spheres

(b) Determination of the $h$-maxima of the distance function for a specified $h$. When the $h$-maxima form a connected component, the algorithm extracts its barycenter.

(c) Random selection of nuclei from the barycenters. The algorithm selects a given barycenter with a specified probability $p$. Each nucleus is then given a nucleation time according to relation (Eq. 19).

(d) The propagation time of each nucleus is computed using the fast marching method through the Python extension module scikit-fmm (Furtney, 2015). The algorithm complexity of the fast marching method is $O(N \ln N)$. The module takes as input the coordinates of the nuclei and a velocity map to compute the distance function. The velocity is equal to one at the locations included in the Boolean spheres and zero otherwise.

(e) The simulated microstructure is obtained from the propagation times according to relation (Eq. 17).

\section{RESULTS AND DISCUSSION}

\section{INTERSTICES SEGMENTATION}

Due to large physical difference between PEEK and copper, the solutions for imaging the microstructure are very restricted. In fact optical microscopic analysis remains the best way to obtain images of satisfactory quality. For instance, scanning Electron Microscope would induce white artefacts and blur around the copper particles. In addition, in our problem, the relatively low resolution of the images makes the selection and the segmentation of the individual particles a rather difficult task. In particular, it is quite difficult to achieve a good segmentation of the PEEK interstices because of the low image resolution and poor grey gradient at the interfaces, so that some of them remain partially replaced by copper. One can see on Fig. 6 that the watershed process achieves particles separation but is not able to include each PEEK layer between them. Once segmented, separated particles in the center of Fig. $6 a$ and Fig. $6 \mathrm{~b}$ do feature an interstice on $6 \mathrm{~d}$ and do not on Fig. 6c, because particles are closer. This phenomenon could be avoided with larger magnification. However it would signify larger images to keep the surface representative, hence more memory and longer processes. One must therefore keep in mind that these phenomenon induces biased assessment of morphological parameters. First, the computation of the perimeter of the copper phase, which remains highly sensitive to interstices presence, must be used carefully. This is mostly the reason which explains why we did not rely on Eq. 14 for the parameter identification phase. Second, the measurements slightly underestimate the contribution of the thinnest interstices to the thickness distribution displayed in Fig. 10.

\section{MODEL}

To estimate the adequation between the model and the experimental images, we considered several morphological criteria, namely the covariance, the granulometry and the thickness distribution of the interstices. The joint use of the covariance and the granulometry is relatively common in morphological models (Jean et al., 2011). The granulometry provides a characterization for the size distribution of the objects that are present in the microstructure, while the covariance provides a second order statistics which characterize the scales present in the microstructure as well as their superimposition. The distribution of interstices is more specific to our model. It is a key parameter of the model: since the conductivity contrast is high between the copper particles and the polymer matrix, large interstices lead to a significant decrease of the electric conductivity of the microstructure at the macroscopic scale.

We show in Fig. 11 a slice of the simulated microstructure obtained with the parameters estimated with the maximum likehood approach. The parameters are reported in 1, with $A_{a}$ the measured surface fraction of copper on segmented images, $\theta$ the intensity of the poisson point process, $\lambda$ and $a$ the parameters of the Gamma distribution of the spheres radii, $h$ the $h$ maxima of the distance function used for selecting the nuclei of the tesselation and $p$ the proportion of $h$ maxima that are randomly selected. The covariance and the granulometry of the simulated and of the experimental microstructures are shown in Fig. 12. A comparison between the thickness distribution of the interstices is displayed in Fig. 13.

Covariances and granulometries taken from simulated and segmented images shows good agreement, assessing the validity of the model. We can observe a small discrepancy, around 0.01 , between the experimental and the simulated covariances for distances ranging from $10 \mu \mathrm{m}$ to $40 \mu \mathrm{m}$. We attribute this discrepancy to the existence of a few large 
Table 1: Model parameters.

\begin{tabular}{ccccccc}
\hline$A_{a}$ & $\theta$ & $\lambda$ & $a$ & $k$ & $h$ & $p$ \\
\hline $63 \%$ & $4.8 \times 10^{-3} \mu \mathrm{m}^{-3}$ & $2.3 \mu \mathrm{m}$ & 0.8 & $1.2 \mu \mathrm{m}$ & $7.26 \mu \mathrm{m}$ & 0.5 \\
\hline
\end{tabular}
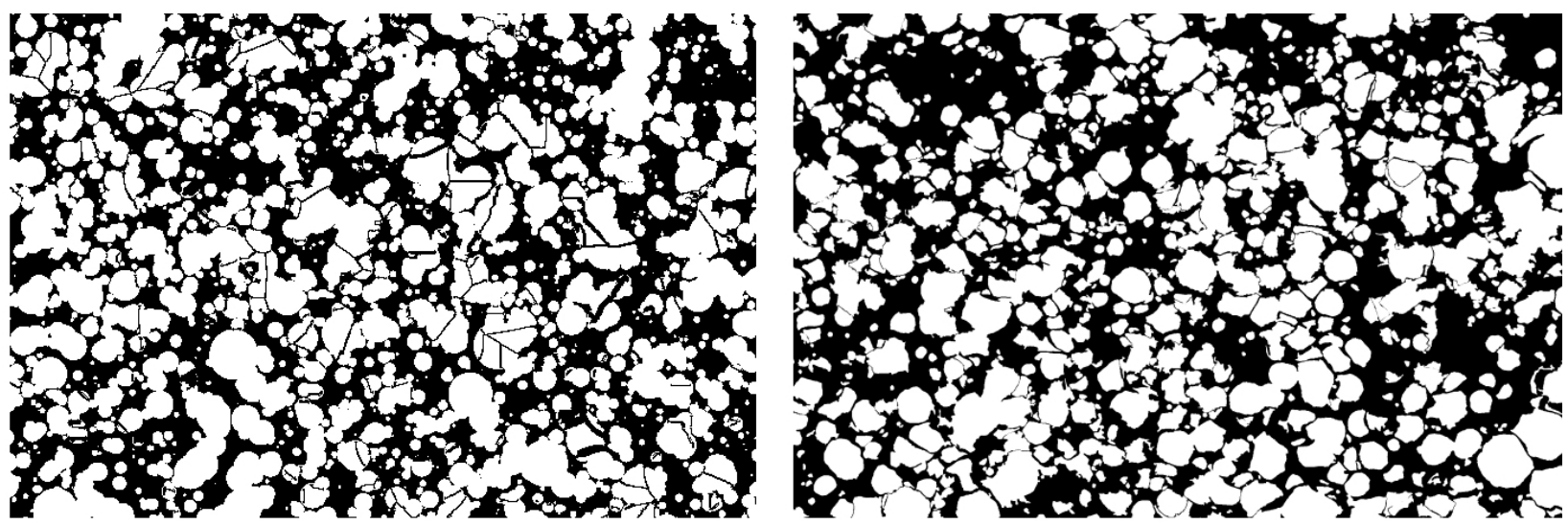

Fig. 11: Simulated microstructure after parameters optimization (left) and experimental microstructure (right), with resolution $0.484 \mu \mathrm{m}$.
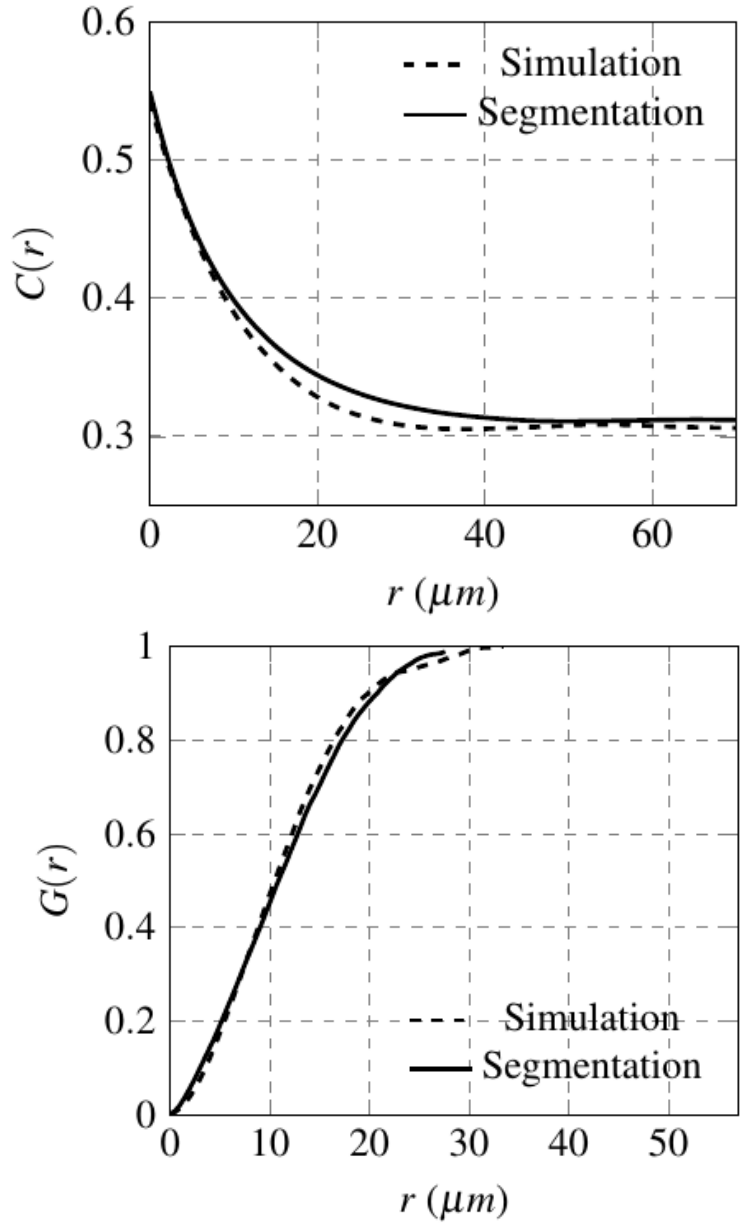

Fig. 12: Simulated and experimental microstructures comparisons between covariances (a) and granulometries (b).

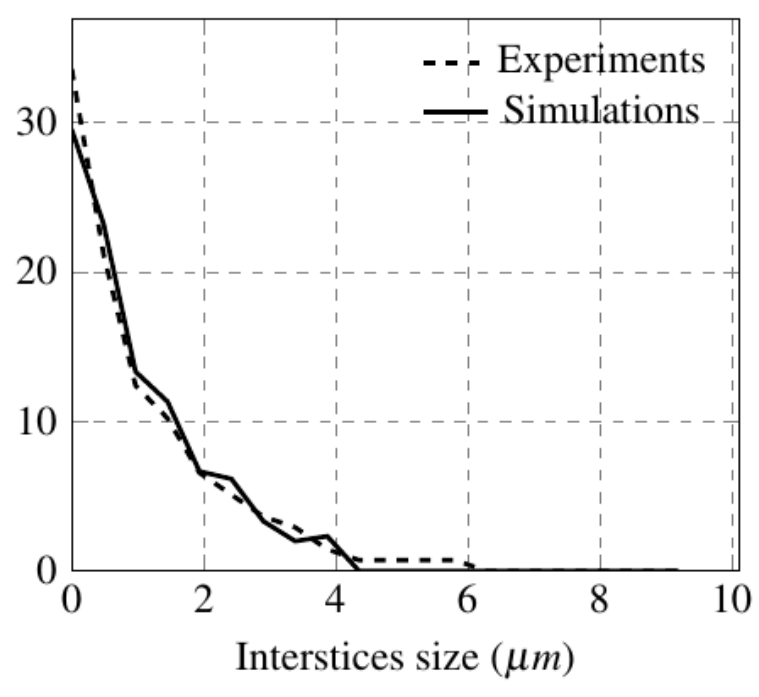

Fig. 13: Comparison between the interstice thickness distribution, as estimated on the simulated and on the experimental images.

zones in the polymer that do not contain copper particles. These large zones can be observed in Fig. 11). To accurately simulate these empty areas, a potential solution is to introduce an additional Boolean model aimed at defining so-called exclusion zones in the microstructure. However, this requires defining additional parameters for describing the model. These parameters are difficult to estimate from the available data: since these empty zones are rarely observed, the experimental data does not provide us with a statistically relevant description of these microstructure areas. In addition, due to their 
scarcity, these zones have little influence on the electric conductivity of the microstructure at the macroscopic scale. Hence, since the covariance curves remain relatively close, we discarded the existence of these empty zones in our model.

\section{CONCLUSION}

In this article, we developed an original method to model the microstructure of a cold spray coating. The two-scale microstructure is modeled by Boolean spheres separated from each other by interstices implanted using a Johnson-Mehl tesselation. The covariance and the granulometry measured on slices of simulated microstructure fit well those from segmented images. The parameters of the model are optimized by fitting distribution law on experimental data and with a Nelder-Mead optimization algorithm. The model allows us to simulate representative volumes of the microstructure. We assessed the representativeness of the model with the covariance, granulometry and also the distribution of interstice size.

The robust and simple segmentation process allow us to use a large amount of images without any complex preparation. The model is flexible enough to easily simulate various shape of particles or volume fraction. Three-dimensional simulation require an fast computation time to generate a small number of representative volumes of coating. These simulated microstructures will eventually allow us to investigate the physical properties of the coating without having to spray a large amount of various coating. This will be done by simulations using finite element method or Fast Fourier Transform.

\section{ACKNOWLEDGEMENTS}

This work was partly carried out within the "C.O.MET" FUI program. The authors would like to thank all the members of the program consortium (under coordination of Dassault Aviation/ArgenteuilFrance) and BPI France, Pôle Astech, Pôle de la céramique, Pôle des microtechniques and DGAC for financial support. BF and FW were supported by grant No FA9550-15-10461 DE from the Air Force Office of Scientific Research (AFOSR).

\section{REFERENCES}

Amsellem O, Madi K, Borit F, Jeulin D, Guipont V, Jeandin M, Boller E, Pauchet F (2008). Two-dimensional (2D) and three-dimensional (3D) analyses of plasma-sprayed alumina microstructures for finite-element simulation of Young's modulus. J Mater Sci 43(12):4091-8.

Beauvais S, Guipont V, Jeandin M, Jeulin D, Robisson A, Saenger R (2008). Study of the porosity in plasma-sprayed alumina through an innovative threedimensional simulation of the coating buildup. Metall Mater Trans A 39(11):2711-24.

Beucher S, Lantuéjoul C (1979). Use of watersheds in contour detection. Proc Int Worksh Image Process RealTime Edge Motion Detect. Rennes, France. Sept 17-21.

Bobzin K, Kopp N, Warda T, Öte M (2012). Determination of the effective properties of thermal spray coatings using $2 \mathrm{D}$ and $3 \mathrm{D}$ models. J Therm Spray Techn 21(6):1269-77.

Chiu SN, Stoyan D, Kendall WS, Mecke J (2013). Stochastic geometry and its applications. Chichester: John Wiley \& Sons.

Cochelin E, Borit F, Frot G, Jeandin M, Decker L, Jeulin D, Al Taweel B, Michaud V, Noel P (1999). Oxidation and particle deposition modeling in plasma spraying of Ti-6Al-4V/SiC fiber composites. J Therm Spray Techn $8(1): 117-24$.

Couka E, Willot F, Jeulin D, Ben Achour M, Chesnaud A, Thorel A (2015). Modeling of the multiscale dispersion of nanoparticles in a hematite coating. J Nanosci Nanotechn 15(5):3515-21.

Delloro F, Jeandin M, Jeulin D, Proudhon H, Faessel M. Bianchi L, Meillot E, Helfen L (2017). A morphological approach to the modeling of the cold spray process. $\mathbf{J}$ Therm Spray Techn 26:1838-50.

Faessel M, Jeulin D (2011). 3D multiscale vectorial simulations of random models. Proc Int Congr Stereol (ICS13). Beijing, China. 19-22.

Faessel M, Bilodeau M (2014). SMIL simple morphological image library. LRDE Tech Rep.

Faessel M (2016). vtkSim software. http://cmm.ensmp.fr/ $\sim$ faessel/vtkSim/demo/

Figliuzzi B, Jeulin D, Faessel M, Willot F, Koishi M, Kowatari N (2016). Modelling the microstructure and the viscoelastic behaviour of carbon black filled rubber materials from 3D simulations. Techn Mech 36:32-56.

Furtney J (2015). Scikit-fmm software. https://github.com/ scikit-fmm

Gasnier JB, Willot F, Trumel H, Figliuzzi B, Jeulin D, Biessy M (2015). A Fourier-based numerical homogenization tool for an explosive material. Matér Tech 103(3):308.

Hermann H (1991). Stochastic models of heterogeneous materials. Zurich: Trans Tech Publ. 
Jean A, Jeulin D, Forest S, Cantournet S, N'guyen F (2011). A multiscale microstructure model of carbon black distribution in rubber. J Microsc 241(3):243-60.

Jeandin M, Borit F, Guipont V, Decker L, Jeulin D, Suzuki M, Sodeoka S (1999). Lattice gas modelling in thermal spraying. Surf Eng, 15(3):191-4.

Jeulin D (2012). Morphology and effective properties of multi-scale random sets: A review. Compt Rend Mécanique 340(4):219-29.

Johnson WA, Mehl RF (1939). Reaction kinetics in processes of nucleation and growth. T Metall Soc AIME 135(8):396-415.

Miles RE (1972). The random division of space. Adv Appl Probab 4:243-66.

El Moumen A, Kanit T, Imad A, El Minor H (2013). Effect of overlapping inclusions on effective elastic properties of composites. Mech Res Commun 53:24-30.

El Moumen A, Imad A, Kanit T, Hilali E, El Minor H (2014). A multiscale approach and microstructure design of the elastic composite behavior reinforced with natural particles. Compos Part B Eng 66:247-54.

Møller J (1989). Random tessellations in $\mathbb{R}^{d}$. Adv Appl Probab 21(1):37-73.

Møller J (1992). Random Johnson-Mehl tessellations. Adv Appl Probab 24:814-44.

Møller J (1994). Lectures on random Voronoi tessellations. Lect Not Stat 87.
Otsu N (1975). A threshold selection method from graylevel histograms. Automatica 11:23-7.

Schneider R, Weil W (2008). Stochastic and integral geometry. Berlin: Springer.

Serra J (1982). Image analysis and mathematical morphology. London: Academic Press.

Sethian JA, Popovici AM (1993). 3-D traveltime computation using the fast marching method. Geophysics 64(2):516-23.

Sethian JA. A fast marching level set method for monotonically advancing fronts (1996). Proc Natl Acad Sci 93(4):1591-5.

Soille P (2003). Morphological image analysis: principles and applications. New York: Springer.

Torquato S (2002). Random heterogeneous materials: microstructure and macroscopic properties. New York: Springer.

Tsitsiklis JN (1995). Efficient algorithms for globally optimal trajectories. IEEE T Autom Contr 40(9):152838.

Vincent L, Soille P (1991). Watersheds in digital spaces: an efficient algorithm based on immersion simulations. IEEE T Pattern Anal 6:583-98.

Zeng QH, Yu AB, Lu GQ (2008). Multiscale modeling and simulation of polymer nanocomposites. Prog Polym Sci 33(2):191-269. 